\title{
Development of Human Breath Acetone Detector Based on Gas Chromatographic Technology
}

\author{
Haiying $\mathrm{Du}^{1,2^{*}}$, Ruizhi Sun ${ }^{1}$, Yanhui Sun ${ }^{2,3}$, Jing Wang ${ }^{2}$, Liang Dong ${ }^{4}$ \\ 1. College of Mechanical and Electronic Engineering, Dalian Minzu University, Dalian 116600, China. \\ 2. School of Electronic Science and Technology, Dalian University of Technology, Dalian 116024,China \\ ${ }^{3}$.College of Information and Communication Engineering, Dalian Minzu University, \\ Dalia116600, China. \\ ${ }^{4}$ Department of Electrical and Computer Engineering, lowa State University, Ames, lowa 50011, USA \\ Corresponding author's e-mail address: duhaiving@dlnu.edu.cn
}

\begin{abstract}
:
Breath acetone is a characteristic biomarker of diabetes. Breath acetone concentration of diabetic patients' breath is higher than that of the normal people. A breath acetone detector based on gas chromatography and semiconductor gas sensor is presented in this paper. The breath collected from diabetic patients is pushed to the gas chromatography separation channel and accelerate flowing by an acceleration pump, then separated using a serpentine gas chromatographic separation channel fabricated by silicon oxide. Carbon dioxide, breath acetone, and ethanol are separated through the stationary phase in the $26^{\text {th }} \mathrm{s}, 162^{\text {th }} \mathrm{s}$ and $324^{\text {th }} \mathrm{s}$, respectively. Separated carbon dioxide, breath acetone, and ethanol will enter the three branches gas detection channel by a time-sharing conversion switch at the end of the serpentine gas chromatographic separation channel, respectively. Then, breath acetone and ethanol were detected by their respective embedded semiconductor gas sensor based on $\mathrm{Y}-\mathrm{Zeolite} / \mathrm{SnO}_{2}$ and $\mathrm{SnO}_{2}$, respectively. The test results show that the method achieves the separation and quantitative detection of carbon dioxide, breath acetone, and ethanol, the separation accuracy is $99.7 \%$ and the accuracy is $99.2 \%$.
\end{abstract}

Key words: Breath acetone, Gas chromatography, Time-sharing conversion switch, Gas sensor

\section{Introduction}

In recent years, with the improvement of people's living standards, advance changes in diet structure and lifestyle have led to various diseases[1]. Diabetes is one of the rapidly increasing diseases. A national survey conducted in 1994, involving 224,251 Chinese residents, 25 to 64 years of age, from 19 provinces, showed that the prevalence of diabetes and impaired glucose tolerance were $2.5 \%$ and $3.2 \%$, respectively[2]. Human breath contains water vapor, carbon dioxide, oxygen, nitrogen, volatile organic compounds (VOCs) produced by metabolism in the body. It shows that a person maybe attacking a disease if the concentration of metabolites produce exceeds a normal range[3]. Breath acetone is the biomarker of diabetes[4]. The concentration of acetone in the exhaled breath of diabetes patients is higher than $1.8 \mathrm{ppm}$, whereas it is lower than $0.8 \mathrm{ppm}$ for those without diabetes[5, 6].

A breath acetone detector based on gas chromatography (GC) and semiconductor gas sensor is reported in this manuscript. The human breath is separated by GC separation channel with time-sharing conversion switch, then the separated breath acetone and ethanol are measured by the gas sensor based on $\mathrm{Y}$ Zeolite/ $\mathrm{SnO}_{2}$ and $\mathrm{SnO}_{2}$. This detector realized the gas separation of breath and quantitative detection of breath acetone in diabetic patients.

\section{The design of the breath acetone detector}

The detector includes three parts: the GC separation channel, gas sensor unit based on semiconductor and SCM detection and display system. The structure principle schematic of breath acetone detector is shown in the Fig.1.

As we can see from the Fig.1 that the GC separation channel is composed of stationary 
phase, GC column, and time-sharing conversion switch. The separated gas is detected quantitatively by gas sensors embedded in different gas channels. The SCM detection and display system is composed of $A / D$ conversion, temperature and humidity detection unit, power supply and LCD display screen.

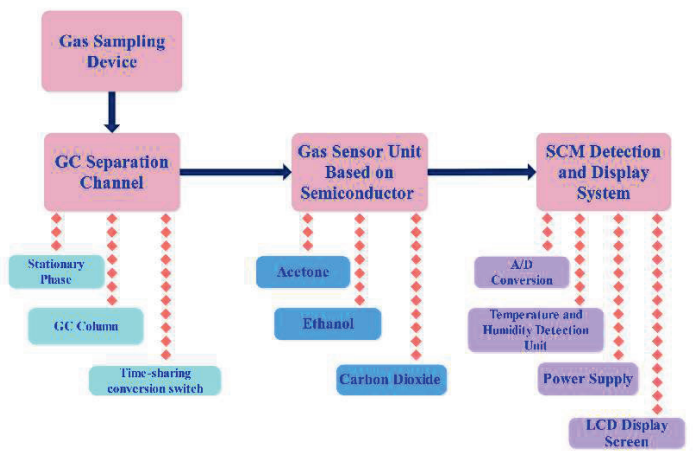

Fig.1. The structure principle schematic of breath acetone detector.

The structure parameters of the GC separation channel are calculated according to Theoretical Plate[7, 8], Rate Theory[9] and pipe inner diameter formula.

The plate number is declared to be 1600 using Formula (1) by Theoretical Plate, where $\mathrm{N}$ is the plate number, $t_{\mathrm{R}}$ is the retention time and $W_{\mathrm{h} / 2}$ is the peak width at half height.

The length is calculated with $2 \mathrm{~m}$ by Formula (2), and (3) according to Rate Theory where $\mathrm{H}$ is the plate height, $L$ is the length, $A$ is the eddy diffusion, $B$ is the longitudinal diffusion, $C$ is the mass transfer resistance and $\bar{v}$ is the average velocity.

And pipe inner diameter is $2 \mathrm{~mm}$ calculated by Formula (4). Where $Q$ is the flow, $V$ is the velocity of the flow. The structure sketch of the GC separation channel is shown in the Fig.2.

$$
\begin{aligned}
& \mathrm{N}=16\left(\frac{t_{R}}{W}\right)^{2}=5.54\left(\frac{t_{R}}{W_{h / 2}}\right) \\
& \mathrm{L}=\mathrm{N} * \mathrm{H} \\
& \mathrm{H}=\mathrm{A}+\frac{B}{\bar{v}}+C \bar{v} \\
& \mathrm{~d}=2 * \sqrt{\frac{Q}{\pi * 3600 V} / 0.001} /
\end{aligned}
$$

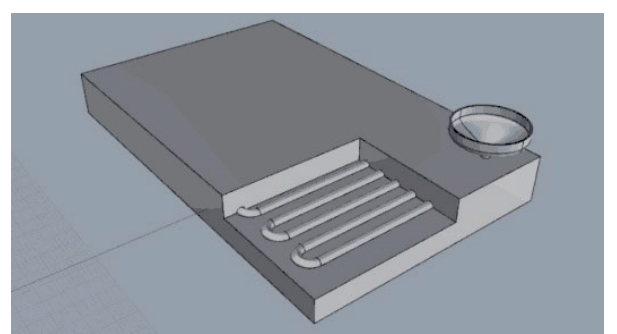

Fig.2. The structure sketch of the GC separation channel.

Gas sensors based on $\mathrm{Y}-\mathrm{Zeolite} / \mathrm{SnO}_{2}$ and $\mathrm{SnO}_{2}$ materials are used to test the concentration of breath acetone and ethanol. The gas sensors are placed at the exit of the channels to detect the contention of breath acetone and ethanol, respectively.

\section{Result and discussion}

The distribution volume fraction of breath acetone is simulated by COMSOL based on the turbulence model and the volume fraction slice diagram of flow phase acetone is shown in the Fig.3.

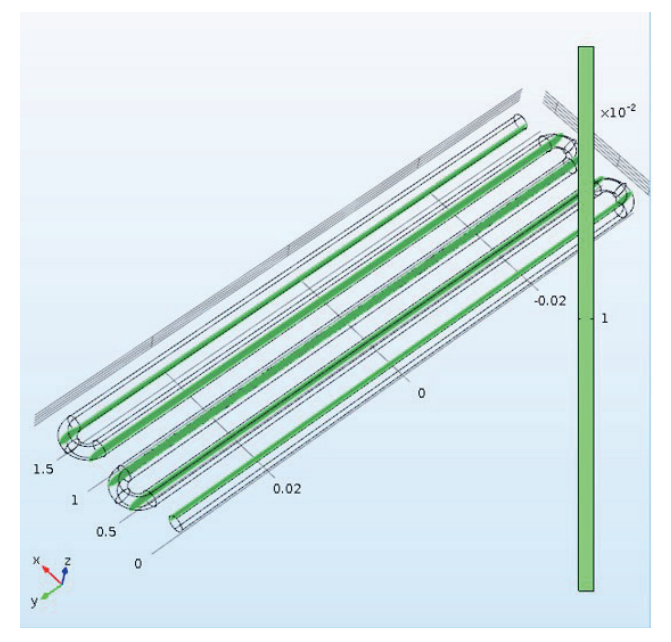

Fig.3. The volume fraction slice diagram of flow phase acetone.

The separation time of carbon dioxide, breath acetone and ethanol are measured by a GC separation channel at the temperature of $40^{\circ} \mathrm{C}$. Carbon dioxide, breath acetone, and ethanol are separated in the $26^{\text {th }} \mathrm{s}, 162^{\text {th }} \mathrm{s}$ and $324^{\text {th }} \mathrm{s}$. Subsequently, a time-sharing conversion switch will operate to convert at $120^{\text {th }} \mathrm{s}$ and $300^{\text {th }} \mathrm{s}$.

Gas sensing properties of $\mathrm{Y}$-Zeolite/ $\mathrm{SnO}_{2}$ sensor and $\mathrm{SnO}_{2}$ sensor are shown in the Fig.4, Fig.5 and Fig.6. It can be seen from the Fig.4 that the optimal operating temperature of $\mathrm{Y}$ Zeolite/ $\mathrm{SnO}_{2}$ sensor and $\mathrm{SnO}_{2}$ sensor are $275^{\circ} \mathrm{C}$ and $200^{\circ} \mathrm{C}$, respectively. Transient responses curves of the $\mathrm{Y}$-Zeolite/ $\mathrm{SnO}_{2}$ sensor to acetone with the concentration of $1 \sim 100 \mathrm{ppm}$ at the operating temperature of $275^{\circ} \mathrm{C}$ are illustrated in 
the curve (a) of the Fig.5. Meanwhile, transient responses curves of the $\mathrm{SnO}_{2}$ sensor to ethanol with the concentration of $1 \sim 100 \mathrm{ppm}$ at the operating temperature of $200^{\circ} \mathrm{C}$ are shown in the curve (b) of the Fig.5. The Fig.6 (a) and (b) show the response times of $\mathrm{Y}$-Zeolite $/ \mathrm{SnO}_{2}$ sensor and $\mathrm{SnO}_{2}$ sensor to acetone and ethanol are the 40 s and 20 s, the recovery times are the 30 s and 40 s, respectively.

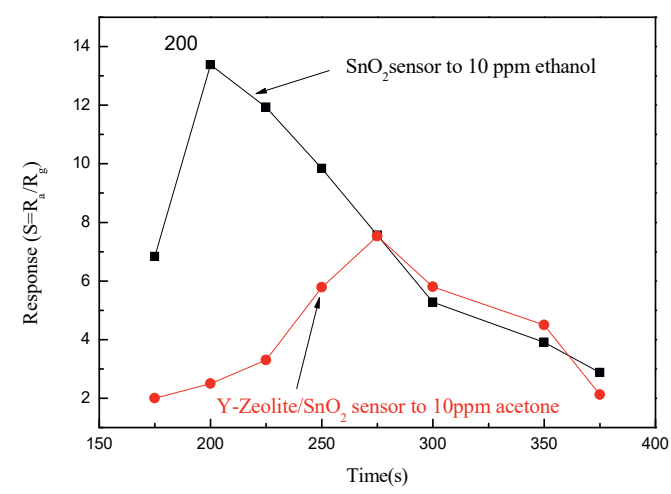

Fig.4. Responses of $\mathrm{Y}$-Zeolite/ $\mathrm{SnO}_{2}$ sensor to 10 ppm acetone and $\mathrm{SnO}_{2}$ sensor to $10 \mathrm{ppm}$ ethanol as a function of operating temperature.

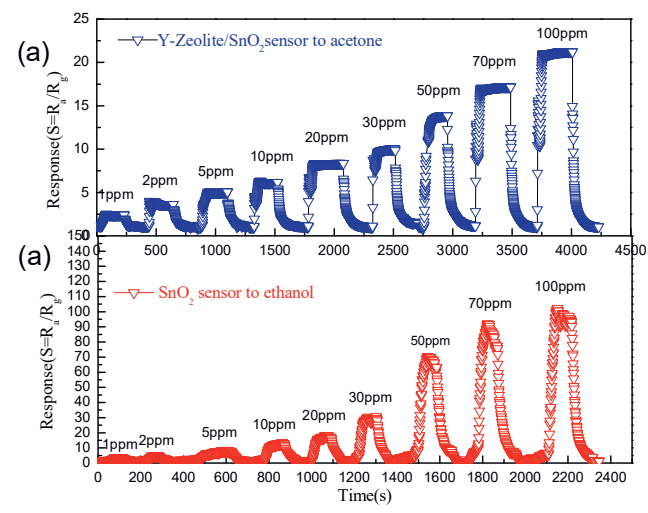

Fig.5. Transient responses curves of $\mathrm{Y}$-Zeolite/ $/ \mathrm{SnO}_{2}$ sensor and $\mathrm{SnO}_{2}$ sensor to acetone and ethanol with the concentration of 1 100 ppm at the operating temperature of $275^{\circ} \mathrm{C}$ and $200^{\circ} \mathrm{C}$.

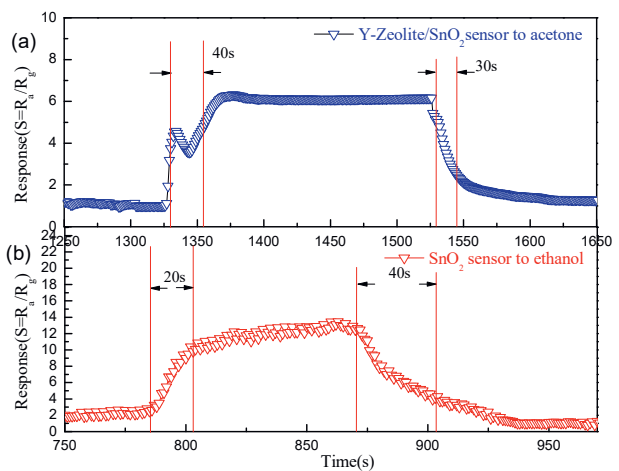

Fig.6. The response and recovery time.

\section{Conclusion}

The human breath acetone detector based on GC separation channel is designed and developed in manuscript. And diabetic patients' breath is sampled and separate effectively by GC separation channel. Carbon dioxide, breath acetone, and ethanol are separated in timesharing conversion switch, the concentration of breath acetone and ethanol are measured by $\mathrm{Y}$ Zeolite/ $\mathrm{SnO}_{2}$ sensor and $\mathrm{SnO}_{2}$ sensor, respectively. Test results show that carbon dioxide, breath acetone, and ethanol of diabetic patients can be separated successfully and measured accurately by the human breath acetone detector. Separated time are 26s for carbon dioxide, 162s for breath acetone and $324 \mathrm{~s}$ for ethanol. The separation accuracy is $99.7 \%$ and the accuracy is $99.2 \%$.

\section{Acknowledgments}

This research was supported in part by the National Natural Science Foundation of China under Grant Number 61501081, 61574025, 11474045, 61501271, the Natural Science Foundation of Liaoning Province under Grant Number 2015020096. L.D. would thank the lowa State's Plant Sciences Institute Faculty Scholar Program for generous support.

\section{References}

[1] THORBURN ALISON N, MACIA L, MACKAY CHARLES R. Diet, Metabolites, and "Western-Lifestyle" Inflammatory Diseases. Immunity, 40(6): 833-42.(2014); doi:

10.1016/j.immuni.2014.05.014

[2] PAN X R, YANG W Y, LI G W, et al. Prevalence of diabetes and its risk factors in China, 1994. National Diabetes Prevention and Control Cooperative Group. Diabetes Care, 20(11): 16649.(1997); doi: 10.2337/diacare.20.11.1664.

[3] AMANN A, POUPART G, TELSER S, et al. Applications of breath gas analysis in medicine. International Journal of Mass Spectrometry, 239(2): 227-33.(2004); doi: 10.1016/j.ijms.2004.08.010.

[4] WANG Z, WANG C. Is breath acetone a biomarker of diabetes? A historical review on breath acetone measurements. Journal of Breath Research, 7(3): 037109.(2013); doi: 10.1088/1752-

7155/7/3/037109.

[5] PARK S. Acetone gas detection using $\mathrm{TiO} 2$ nanoparticles functionalized In2O3 nanowires for diagnosis of diabetes. Journal of Alloys \& Compounds, 696(2016); doi:

10.1016/j.jallcom.2016.11.298.

[6] WEI S, ZHAO G, DU W, et al. Synthesis and excellent acetone sensing properties of porous WO 3 nanofibers. Vacuum, 124(32-9.(2016); doi: 10.1016/j.vacuum.2015.11.010.

[7] GIDDINGS J C. Dynamics of Chromatography: Principles and Theory. Crc Press, 2002);

[8] MORETTI P, VEZZANI S, CASTELLO G. Prediction of theoretical plate number in isothermal gas chromatographic analysis on capillary columns. 
Journal of Chromatography A, 1133(1): 305-

14.(2006); doi: 10.1016/j.chroma.2006.08.023.

[9] HAWKES S J. Modernization of the van

Deemter equation for chromatographic zone

dispersion. Journal of Chemical Education, 60(5):

393-8.(1983); doi: 10.1021/ed060p393. 